\title{
Effects of Single versus Multiple Bouts of Resistance Training on Maximal Strength and Anaerobic Performance
}

\author{
by \\ Kai Shiau', Te Hung Tsao², Chang Bin Yang 3
}

\begin{abstract}
This study examined the effects of one single bout daily versus triple bouts of resistance exercise for 12 weeks on muscular strength and anaerobic performance of the upper body. Twenty young male adults (age: $22.0 \pm 1.0$ years, bench press: $44.0 \pm 10.3 \mathrm{~kg}$ ) were randomly assigned to a single bout (SB) or triple bouts (TB) of resistance exercise group. Maximal strength and anaerobic performance of the upper body using the bench press (one-repetition maximum) and the modified $30 \mathrm{~s}$ Wingate test were determined before and after the intervention. Additionally, changes in lactate levels before and after the Wingate test were measured. Although the SB and TB groups showed a significant increase in maximal strength (post-intervention, $S B: 67.2 \pm 9.2$ and TB: $67.6 \pm 7.6 \mathrm{~kg}$, respectively) compared with the values at pre-intervention (SB: $44.6 \pm 11.4$ and TB: $43.9 \pm 8.7 \mathrm{~kg}$, respectively), there was no significant difference for this variable between the two groups post-intervention $(p>0.05)$. The anaerobic performance of the upper body in the $S B$ and TB groups also displayed improvements without significant difference between the two groups after the completion of different training regimes. On the basis of the same training volume, multiple bouts of resistance training showed similar improvements in maximal strength and anaerobic performance to one bout of resistance training in young adult men without prior experience in resistance training
\end{abstract}

Key words: muscular performance, Wingate test, training frequency.

\section{Introduction}

The American College of Sports Medicine (ACSM) and the American Heart Association (AHA) jointly recommend that adults need a minimum of 30 minutes of moderate-intensity aerobic physical activity 5 days a week or a minimum of 20 minutes of vigorous aerobic activity 3 days a week to promote and maintain health (Haskell et al., 2007). However, busy schedules often present obstacles to finding sufficient time to exercise (Reichert et al., 2007). To counter this, multiple bouts of aerobic exercises or physical activities totaling 30 minutes or longer are an alternative recommended regime (Haskell et al., 2007; Tremblay et al., 2011). Regarding the effect of multiple bouts vs. one bout of aerobic exercise, several studies have reported that multiple bouts versus one single bout of aerobic exercise, for example brisk walking or walking and running, displayed comparable improvements in cardiopolumary fitness (Macfarlane et al., 2006), blood pressure (Serwe et al., 2011), blood glucose and insulin resistance (Baynard et al., 2005) as well as blood lipids (Miyashita et al., 2008) on the basis of the same exercise duration or volume. However, researchers have emphasized that the minimum duration for a single session in a multiple-bout of aerobic exercise should be at least 10 minutes (Miyashita et al., 2008; O'Donovan et al., 2010).

On the other hand, resistance exercise using major muscle groups with 8-10 exercises for two or more nonconsecutive days per week is also

1 - Air Force Institute of Technology, Kaohsiung, Taiwan.

2 - Physical Education Section of General Education, National Sun Yat-Sen University, Kaohsiung, Taiwan.

3 - Department of Physical Education and Kinesiology, National Dong Hwa University, Hualien, Taiwan. 
recommended for adults as muscular strength and endurance (Fitzgerald et al., 2004; LiuAmbrose et al., 2010), bone density (Bemben et al., 2011; Vanni et al., 2010) and insulin phenomenon (Ng et al., 2011) can be improved by a regular resistance or weight training program. In addition to the aforementioned merits, anaerobic performance was enhanced after regular resistance training (Fatouros et al., 2005; Santos and Janeira, 2012).

Considering the aspect of muscular performance, multiple sets or sessions of resistance training were superior to one single set or session of resistance training because of more training volume or loading (Bottaro et al., 2011; Krieger, 2010). However, in contrast to the abovementioned studies regarding multiple bouts of aerobic exercise and dose-response from multiple sets vs. one single set of resistance exercise, only few studies have examined whether there was any difference for muscular strength performance and anaerobic capacity between one single bout and multiple bouts of resistance exercise with equal training volume.

Therefore, the aim of this study was to investigate the effects of one single bout versus triple bouts of resistance exercise on muscular performance of the upper body. In addition, the difference in anaerobic capacity (Wingate anaerobic test, WAnT) and lactate response to the WAnT due to these two different resistance training regimes were investigated in this study. Given an equal total training volume (loading and sets) in the two resistance training regimes, the hypothesis of this study was that the improvements in maximal strength would be similar between these two different regimes.

\section{Methods}

\section{Participants and experimental overview}

Eligible participants who were juniors in a military college and had no experience in resistance training qualified for the study. Although the subjects did not participate in any structured training, they had 3-4 times of extracurricular ball sports such as basketball, volleyball, table tennis and soccer per week, approximately $30 \mathrm{~min}$ per session. All participants (age: $22.0 \pm 1.0$ years, body height: $170.5 \pm 6.2 \mathrm{~cm}$, body mass: $68.2 \pm 5.3 \mathrm{~kg}$ ) were given an initial medical and physical examination and were excluded if they had metabolic, cardiovascular or musculoskeletal diseases, or ingested any medications, anabolic steroids or nutritional supplements known to affect resistance exercise performance or muscle protein synthesis at least one year before this study. During the study period, the aforementioned substances were not allowed either. The sample size was determined using power analysis software G*Power 3.0 (Franz Faul, Kiel University, Germany), on the basis of the results of the maximal bench press performance in Baker et al. (2013). Accordingly, each group should comprise 10 participants to provide $80 \%$ power at the 0.05 level of significance. All participants (male, age $=22.0 \pm$ 1.0 years) were randomly assigned to one of the two following groups: one single bout (SB) or triple bouts (TB) group. Before the intervention, each participant's maximal strength (bench press) was determined; furthermore, the upper body Wingate test was applied and changes in lactate concentration from pre- to $30 \mathrm{~min}$ post-Wingate test performance were measured. All the assessments were repeated after the 12-week resistance training intervention. The subjects were adequately informed about the risks and benefits involved in the study and provided written informed consent to participants. This study was approved by the institutional review board of the Ani Tai Hospital, Taiwan.

\section{Anthropometry}

Body height and mass of all participants were measured with an electronic stadiometer (Seca, Model 242, Hanover, MD) and digital scales (Tanita, Model BWB-627A, Hong Kong, China), respectively. The body mass index (BMI) was calculated by dividing body mass $(\mathrm{kg})$ by body height $(\mathrm{m})$ squared $\left(\mathrm{kg} / \mathrm{m}^{2}\right)$. In addition, the body fat percentage $(\%)$ and fat-free mass $(\mathrm{kg})$ were measured using an InBody 720 analyzer (BIOSPACE, Seoul, South Korea). The measurements were performed in the morning after an overnight fast.

\section{Maximal Strength Measurement}

The one-repetition maximum (1-RM) bench press was used for the maximal strength assessment of the upper body. It was determined by three to six sets with $2 \mathrm{~min}$ rest intervals between sets. The pre- and post-training assessments were scheduled at least $24 \mathrm{~h}$ before the first training session and after the final 
training session, respectively. After a 3-5 min warm-up, participants started their maximal bench press evaluation. The participant lowered the bar to the mid-chest and then pressed the weight until the elbows were fully extended for at least 3 s. Pre- and post-assessments were scheduled at the same time of the day in order to limit confounding variables.

\section{The Wingate Test}

Before and after the resistance training program, an arm WAnT for upper body power was arranged approximately one hour after the maximal strength assessment. A Monark 824E ergometer was modified with an arm crank and the resistance load was equal to $0.05 \mathrm{kp} / \mathrm{kg}$ body mass. After a 2 min warm-up with no load, participants were signaled to crank for $30 \mathrm{~s}$ as rapidly as they could. The peak power output $(\mathrm{PP})$ during any $5 \mathrm{~s}$ period, the average power output (AP) for total $30 \mathrm{~s}$, and the fatigue index (FI) were calculated according to standard equations (Inbar et al., 1996). Verbal encouragement was provided during this testing period.

\section{Lactate Measurement}

Immediately before and after the WAnT, and at the $3^{\text {rd }}, 5^{\text {th }}, 15^{\text {th }}$, and $30^{\text {th }} \mathrm{min}$ post-WAnT, participants' finger capillary blood samples were collected for the lactate analysis using a Lactate Pro analyzer (Arkray, Shiga, Japan). Before taking the blood sample, participants immersed one hand in warm water $\left(\sim 40^{\circ} \mathrm{C}\right)$ for $30 \mathrm{~s}$. The hand was dried and then one finger was pierced with a sterile lancet $(1.5 \mathrm{~mm})$. The first drop of blood was removed and $\sim 10 \mu \mathrm{L}$ of blood was used to determine lactate concentrations. For the lactate concentrations after the WAnT, there were five collection time points. The first three (immediately, 3 and $5 \mathrm{~min}$ after the WAnT) and last two points ( 15 and $30 \mathrm{~min}$ after the WAnT) represented the effectiveness of the anaerobic and aerobic energy systems, respectively (Gleeson, 1996; Rimaud et al., 2010). The lactate measurements were also scheduled before and after the resistance training program.

\section{Training Program}

All participants performed the resistance training program on three nonconsecutive days per week for 12 weeks. The training exercises consisted of the lateral raise, lateral pull-down, shoulder press, biceps curl, triceps extension, pectoral fly, abdominal crunch, back extension and abdominal crunch exercises. All exercises were performed on resistance equipment (Johnson, Taipei, Taiwan) and consisted of three sets for each exercise. The loadings and repetitions during the W1-W4, W5-W8, and W9W12 were $50-55 \%$ of $1 \mathrm{RM}$ and $12-15$ repetitions, $65 \%$ of $1 \mathrm{RM}$ and 10 repetitions, and $75 \%$ of $1 \mathrm{RM}$ and 8 repetitions, respectively. In the SB group, participants engaged in their training program at $5 \mathrm{pm}$ and finished three sets of one exercise before starting the next exercise. The rest intervals between sets and exercises were 30 and $60 \mathrm{~s}$, respectively. In the TB group, each bout was performed at $8 \mathrm{am}, 5 \mathrm{pm}$ and $9 \mathrm{pm}$, respectively. The participants performed only one set for all exercises in each bout and the rest time between exercises equaled $30 \mathrm{~s}$. Because the minimum period for each bout of resistance training in the multiple-bout regime had not been previously determined, $10 \mathrm{~min}$ for each bout in the TB regime was established by referring to the model of daily multiple exercise bouts in previous studies (Miyashita et al., 2008; O'Donovan et al., 2010).

Diet logs were collected before the initiation of the training program, at the $6^{\text {th }}$ and $12^{\text {th }}$ week. Each collection comprised a 3 day period, including one weekend day and two weekdays. In addition, the diet instruction sessions were scheduled twice, the first time at one week before the start of the training program and the other time at the $6^{\text {th }}$ week. In the diet instruction sessions, a nutritionist instructed the participants how to recognize and record food categories and portions. Diet software (DietOrganizer 2.2, MulBerrySoft, USA) was used for analyses of total calories and nutrient composition. On the other hand, participants were required to maintain their habitual physical activities. However, the physical activities in each week during the experiment were categorized as two parts: weekdays and weekends. On the basis of the physical activity record (International Physical Activity Questionnaire (IPAQ) - short form, Craig et al., 2003), most of the physical activities during the weekdays were similar as the subjects were students of the same military college. During the weekends, although all participants could engage in any activities freely, no resistance or weightlifting exercise was allowed. The results regarding daily physical activity did not show any 
significant difference between the two groups.

\section{Statistical analysis}

All data were expressed as means \pm standard deviation (SD). The Kolmogorov-Smirnov method was used to test for normality of the data $(p>.05)$. The variables were analyzed using a two-way mixed-design analysis of variance (ANOVA) (group $\times$ time) followed by post hoc comparisons. The associations between the variables were performed by the Pearson product-moment correlation coefficient. The statistical significance was set at $p<.05$. The statistical procedures were conducted using SPSS (version 16, Chicago, IL, USA).

\section{Results}

The BMI and body composition of the two groups are presented in Table 1. No significant differences were evident between the two groups in terms of the BMI, body fat percentage or fatfree mass before the training program began $(p>$ 0.05). On completion of the training program, although both of the two groups displayed a slight decrease in the body fat percentage, no significant differences were observed either within the same group or between the two groups $(p>0.05)$. On the other hand, the fat-free mass at post-intervention showed significantly higher values in the SB (pre: $62.0 \pm 6.3$ and post: $64.3 \pm 5.9$ $\mathrm{kg}, p<0.05$ ) and TB (pre: $62.3 \pm 6.0$ and post: $64.7 \pm$ $6.0 \mathrm{~kg}, p=0.021)$ compared with pre-intervention by $11 \%$ and $12 \%$, respectively. However, the fatfree mass after the completion of different training regimes did not significantly differ between the two groups $(p>0.05)$.

As for the diet log results, the average daily intake of calories for the SB and TB groups was $2500 \pm 224$ versus $2484 \pm 301,2737 \pm 204$ versus $2648 \pm 313$, and $2897 \pm 263$ versus $2793 \pm 288 \mathrm{kcal}$ at the start, middle and end of the resistance training program, respectively. Although the average daily calorie intake showed no significant difference between the two groups at the three measured points, a gradually increasing trend for food intake was visible for each group between the beginning and the end of the training program and the mean total caloric consumption per day was significantly greater for each group $(p<0.05)$ at the $12^{\text {th }}$ week compared to the values at the beginning of the experiment.
Maximal strength assessment and the Wingate Test

The results of the bench press and Wingate test are displayed in Table 2. Although the results for bench press in the SB (pre: $44.6 \pm 11.4 \mathrm{~kg}$ and post: $67.2 \pm 9.2 \mathrm{~kg}$, respectively; $p<0.05$ ) and TB (pre: $43.9 \pm 8.7 \mathrm{~kg}$ and post: $67.6 \pm 7.6 \mathrm{~kg}$, respectively; $p<0.05$ ) groups were significantly higher compared with the values at preintervention by $33 \%$ and $37 \%$, the maximal strength at the post-intervention between the two groups did not show a significant difference.

The Wingate test showed no significant difference in $\mathrm{PP}, \mathrm{AP}$, or FI between the SB and $\mathrm{TB}$ groups before the training program. After resistance training, the $\mathrm{PP}, \mathrm{AP}$, and $\mathrm{FI}$ in the $\mathrm{SB}$ and TB groups showed significantly higher values than at pre-intervention by $15 \%$ and $19 \%, 11 \%$ and $9 \%, 28 \%$ and $22 \%$, respectively. However, these three variables of the Wingate test did not show any significant differences between the two groups after different resistance training protocols. Lactate

Table 3 shows the lactate results at each measured point during the Wingate test and the area under the curve for lactate $\left(A \mathrm{AC}_{\mathrm{L}}\right)$ across all measured points for both groups before and after the intervention. Although the lactate levels of the two groups were significantly higher immediately after the Wingate test and at the $3^{\text {rd }}, 5^{\text {th }}$, and $15^{\text {th }}$ min post-Wingate test compared with preWingate test before and after the intervention of resistance training $(p<0.05)$, there was no significant difference for each measured time point between different training regimes $(p>0.05)$. In addition, the AUCL did not show a significant difference between the groups before or after each training program, either.

With respect to the relationship between the variables in this study, the changes in fat-free mass were significantly associated with the changes in maximal strength $(r=0.81$ and 0.80 in the SB and TB groups, respectively, $p<0.05$ ) and the Wingate test $(r=0.78$ and 0.75 for the PP in the SB and TB groups, respectively; $r=0.72$ and 0.78 for the AP in the SB and TB groups, respectively; $r$ $=0.60$ for the FI in the SB group, all $p<0.05$ ). On the other hand, no significant relationships were found between the changes in the body fat percentage and the changes in maximal strength or the Wingate test. 
Table 1

Age and body composition of the $S B$ and $T B$ groups at pre- and post-training intervention

\begin{tabular}{ccccc}
$\mathrm{SB}(\mathrm{n}=10)$ & \multicolumn{2}{c}{$\mathrm{TB}(\mathrm{n}=10)$} & & \\
pre $\quad$ post & pre $\quad$ post & Within & $\begin{array}{c}\text { Between } \\
\text { group }\end{array}$
\end{tabular}

\begin{tabular}{ccccccc}
\hline Age (years) & $22.0 \pm$ & \multicolumn{5}{c}{$21.9 \pm$} \\
& 1.1 & & 1.0 & $\times$ & $\times$ \\
BMI & $24.1 \pm$ & $24.3 \pm$ & $24.4 \pm$ & $24.4 \pm$ & $\times$ & $\times$ \\
$\left(\mathrm{kg} / \mathrm{m}^{2}\right)$ & 1.0 & 1.0 & 1.1 & 0.9 & & \\
Body fat $\%$ & $17.2 \pm$ & $16.6 \pm$ & $17.0 \pm$ & $16.4 \pm$ & $\times$ & $\times$ \\
& 2.1 & 2.0 & 2.3 & 2.1 & & \\
FFM $(\mathrm{kg})$ & $62.0 \pm$ & $64.3 \pm$ & $62.3 \pm$ & $64.7 \pm$ & SB: $p<0.05$, & $\times$ \\
& 6.3 & 5.9 & 6.0 & 6.0 & TB: $p=$ & \\
& & & & & 0.021 &
\end{tabular}

Data are mean $\pm S D, \times$ means $p>0.05$

BMI: body mass index; FFM: fat free mass.

Table 2

Pre and post-intervention results of the bench press and Wingate test for both groups

SB $(\mathrm{n}=10) \quad$ TB $(\mathrm{n}=10)$

pre post pre post Within Between

group groups

\begin{tabular}{ccccccc}
\hline Maximal & $44.6 \pm$ & $67.2 \pm$ & $43.9 \pm 8.7$ & $67.6 \pm$ & $p<0.05$ & \\
strength & 11.4 & 9.2 & & 7.6 & \\
& & & & & \\
& & & & & \\
Wingate & $843.9 \pm$ & $857.1 \pm$ & $833.3 \pm$ & $876.7 \pm$ & $p<0.05$ & \\
& 128.9 & 101.8 & 126.5 & 104.1 & & $\times$ \\
PP & & & & & \\
& & & & & \\
& & & & & \\
AP & $429.2 \pm$ & $448.7 \pm$ & $426.0 \pm$ & $444.8 \pm$ & $p<0.05$ & \\
& 43.6 & 35.0 & 38.1 & 49.3 & \\
& & & & & \\
FI & $69.7 \pm 7.2$ & $72.2 \pm$ & $67.8 \pm 5.7$ & $70.2 \pm$ & $p<0.05$ & \\
& & 6.9 & & 5.6 & \\
\hline
\end{tabular}

Data are mean $\pm S D, x$ means $p>0.05$.

PP: peak power, AP: average power, FI: fatigue index; Units: $\mathrm{kg}$ for the maximal strength, watt (W) for AP and PP, \% for FI. 


\begin{tabular}{|c|c|c|c|c|c|}
\hline \multirow{2}{*}{\multicolumn{6}{|c|}{$\begin{array}{c}\text { Table } 3 \\
\text { Changes in lactate concentration pre and post Wingate test before and after the intervention }\end{array}$}} \\
\hline & & & & & \\
\hline & \multicolumn{2}{|c|}{ Before Intervention } & \multicolumn{2}{|c|}{ After Intervention } & \multirow[t]{2}{*}{$p$} \\
\hline & $\mathrm{SB}(\mathrm{n}=10)$ & $\mathrm{TB}(\mathrm{n}=10)$ & $\mathrm{SB}(\mathrm{n}=10)$ & $\mathrm{TB}(\mathrm{n}=10)$ & \\
\hline Pre & $1.5 \pm 0.3$ & $1.5 \pm 0.4$ & $1.5 \pm 0.3$ & $1.5 \pm 0.4$ & $x$ \\
\hline Imm Post-* & $6.8 \pm 2.5$ & $6.1 \pm 2.8$ & $6.1 \pm 2.3$ & $6.0 \pm 2.2$ & $x$ \\
\hline 3 min Post- * & $12.7 \pm 6.8$ & $13.4 \pm 6.1$ & $12.6 \pm 6.5$ & $13.0 \pm 6.0$ & $x$ \\
\hline 5 min Post- * & $9.3 \pm 3.1$ & $9.2 \pm 2.8$ & $8.9 \pm 2.3$ & $9.0 \pm 2.2$ & $x$ \\
\hline 15 min Post- * & $4.6 \pm 1.1$ & $4.2 \pm 1.7$ & $4.3 \pm 1.0$ & $4.3 \pm 1.2$ & $x$ \\
\hline 30 min Post- & $2.0 \pm 1.1$ & $2.0 \pm 1.1$ & $1.9 \pm 0.8$ & $1.9 \pm 0.9$ & $x$ \\
\hline$A U C_{L}$ & $98.1 \pm 8.2$ & $101.7 \pm 7.9$ & $97.2 \pm 8.0$ & $100.0 \pm 7.7$ & $x$ \\
\hline \multicolumn{6}{|c|}{$\begin{array}{l}\text { Data are mean } \pm S D, \times \text { means } p>0.05 \text { between the } S B \text { and TB groups. } \\
\text { Unit: } m \text { mol/L; }{ }^{*} \text { means significantly different from pre- and } 30 \text { min post. Pre: pre-Wingate test, } \\
\text { Imm Post-: immediately post-Wingate. } A U C_{L} \text { : the area under the curve of lactate }\end{array}$} \\
\hline
\end{tabular}

\section{Discussion}

Only few studies have examined the effect of long-term one single bout versus multiple bouts of resistance exercise on maximal strength or anaerobic capacity of the upper body. The current study shows that multiple bouts of resistance exercise (TB) vs. a traditional one bout of resistance training (SB), with equal training volume (sets and loads), elicited corresponding improvements in maximal strength and anaerobic performance of the upper body. For those who are unable to tolerate high loading or volume in one bout of resistance training, dispersed bouts of resistance training can result in similar improvements in fat-free mass and muscle performance to one bout of resistance training.

\section{Body Composition}

Before and after the intervention, the body fat percentage and BMI did not significantly differ between-group or within-group. On the other hand, although the fat-free mass in both groups showed a significant increase at postintervention compared with the values at preintervention, there were no significant differences in this variable between the two groups after the training program. The former result was similar to the findings in the studies of Rabelo et al. (2011) and Washburn et al. (2012) which identified a significant increase in fat-free mass in elderly women and young adults after a resistance training intervention, respectively. Of the several variables affecting body composition, diet and training are the two of factors observed during the intervention. After analyzing participants' diet logs, it was found that the calorific intake of both groups was significantly higher at postintervention than at pre-intervention, although this variable did not show a significant difference between the studied groups at the three measured points. On the other hand, Candow and Burke (2007) concluded that training volume, frequency, intensity (sets and loading) and duration might 
have influence on the changes in fat-free mass and strength performance due to resistance training intervention. Therefore, the observed increases in average daily calorie intake and training volume likely produced a synergistic effect leading to an increased fat free mass in this study, however, the similar gradual increases in training volume and calorie intake between the two groups might be the reasons for the comparable change in fat free mass between them.

\section{Maximal Strength}

It has been reported that the primary outcome of resistance training intervention is the enhancement of muscular strength performance (Fitzgerald et al., 2004; Liu-Ambrose et al., 2010). In addition to similar findings to the aforementioned studies and in support for the hypothesis of this study, our results show that changes in fat free mass were significantly related to changes in maximal strength (bench press). The literature (Hunter et al., 2004; Rabelo et al., 2011; Reeves et al., 2004) suggests that an increase in fat free mass due to resistance training intervention could contribute to an enhancement in maximal strength. On the basis of the findings of this study, an increase in fat free mass resulting from resistance training programs might be a factor contributing to the enhancement of maximal strength. On the other hand, there was a comparable improvement in maximal strength found in both groups. This study is the first to compare the results between one single bout versus multiple bouts of resistance exercise and there was little data available for reference. One study (Moraes et al., 2013) reported that different resistance programs with similar training volume could lead to similar enhancement in maximal strength in untrained adolescents. As a result, this study deduced that equal training volume, the same loading and total sets, might play a role in strength gains. Yet, neuromuscular adaptions can also contribute to improvement in maximal muscular strength (Blazevich et al., 2007). This is a limitation of our study as we did not perform any measurements of neural control. Therefore, although the results concerning maximal strength supported the hypothesis of this study, further research regarding changes in neural control as well as muscle mass is necessary.

\section{Anaerobic Performance}

In addition to maximal strength, resistance training intervention can enhance anaerobic sport performance, measured in terms of the Wingate test, vertical jump, and countermovement jump (Fatouros et al., 2005; Santos and Janeira, 2012; Slade et al., 2002). In this study, comparable improvements in the Wingate test variables (PP, AP and FI) were found in both groups. However, previous studies had utilized the Wingate test for the lower body as opposed to the upper body. Hence, the current study adds the evidence that resistance training generates improvement in anaerobic performance of the upper body as well. Several authors (Vardar et al., 2007; Kim et al., 2011) have concluded that an increase in fat free mass due to resistance training was related to the improvement in anaerobic performance. Significant correlations between the changes in the fat free mass and most variables of the Wingate test in both groups also occurred in the present study. As a result, the increased fat free mass might be one of the factors contributing to the improvements of anaerobic performance as well as maximal strength of the upper body in this study. With respect to the changes in lactate levels from baseline to $30 \mathrm{~min}$ post-Wingate test, although the lactate levels did significantly increase after the Wingate test, there were no significant differences in lactate levels for each measured point between the SB and TB groups. Furthermore, there were no significant differences for the AUCL before and after 12 weeks of resistance training, either. Several studies (Chromiak et al., 2004; MacDougall et al., 1977) have suggested that the enhancement of the anaerobic energy system is one possible factor responsible for the improvement in anaerobic sport performance due to resistance training intervention. Beneke et al. (2002) indicated that the energy supply proportions of the aerobic system, phosphate system, and lactic acid system in the Wingate test were $18.6 \%, 31.3 \%$, and $50.3 \%$, respectively. This study found that the lactate levels on completion of the intervention were lower at each post-Wingate test time point compared with the same measured point before the resistance training intervention, although no significant difference was found between the groups. Thus, this study infers that the lactate levels may be affected by resistance training intervention via enhancing lactate clearance from these trivial differences. On the other hand, the 
effectiveness of the anaerobic and aerobic energy systems might be similar as lactate concentrations at each post-WAnT measured point did not show a significant difference between the two different resistance training regimes. However, comparable effectiveness in the energy system of the WAnT was our speculation, because the energy systems were not considered in this study. As a result, the energy systems and related enzymes deserve further research when comparing one single bout versus multiple bouts of resistance exercise in the future.

\section{Limitations}

Although the total time spent on resistance exercises for each day was equal between the SB and TB groups, there was an obvious difference in terms of the rest time within each bout between the two groups (SB: $~ 17 \mathrm{~min}$ and TB: $\sim 4 \mathrm{~min}$, respectively). Several studies (Oliveira et al., 2008; Villanueva et al., 2015) have indicated that different rest intervals between resistance exercises or sets might affect muscle performance. However, those studies utilized the protocol of multiple sets in one single bout of resistance exercise, similar to the SB in this study. Secondly, those studies focused on the effect of rest between exercises on sport performance and did not measure anaerobic performance or lactate metabolism. Thus, caution should be taken when generalizing our results. In addition, given that the gains in muscular strength and the Wingate test were attained in a population of young adults without any experience in resistance training, these results may not be generalized to other populations such as elite athletes, experienced weight lifters or professional bodybuilders. Therefore, those populations should be further examined in the future.

In conclusion, the current study demonstrates that multiple bouts of resistance exercise resulted in similar improvements in maximal strength and anaerobic performance compared to one bout of resistance exercise. These findings indicate comparable efficacy observed between one single bout versus multiple bouts of aerobic exercise. The increase in fat free mass resulting from the intervention of different resistance training regimes with equal training loading may contribute to the improvements in maximal strength and anaerobic performance. However, further research is warranted regarding potential alterations in neural control and energy systems caused by multiple bouts of resistance training.

\section{Acknowledgements}

The authors sincerely appreciated Dr. Naokata Ishii's (The University of Tokyo) instructions and opinions on resistance training and manuscript writing.

\section{References}

Baker JS, Davies B, Cooper SM, Wong DP, Buchan DS, Kilgore L. Strength and body composition changes in recreationally strength-trained individuals: Comparison of one versus three sets resistance-training programmes. BioMed Res Int, 2013; 2013: 1-6

Baynard T, Franklin RM, Goulopoulou S, Carhart R Jr, Kanaley JA. Effect of a single vs multiple bouts of exercise on glucose control in women with type 2 diabetes. Metabolism, 2005; 54: 989-994

Bemben DA, Bemben MG. Dose-response effect of 40 weeks of resistance training on bone mineral density in older adults. Osteoporos Int, 2011; 22: 179-186

Beneke R, Pollmann C, Bleif I, Leithäuser RM, Hütler M. How anaerobic is the Wingate Anaerobic Test for humans? Eur J Appl Physiol, 2002; 87: 388-392

Blazevich AJ, Gill ND, Deans N, Zhou S. Lack of human muscle architectural adaptation after short-term strength training. Muscle Nerve, 2007; 35: 78-86

Bottaro M, Veloso J, Wagner D, Gentil P. Resistance training for strength and muscle thickness: Effect of number of sets and muscle group trained. Sci Sports, 2011; 26: 259-264

Candow DG, Burke DG. Effect of short-term equal-volume resistance training with different workout frequency on muscle mass and strength in untrained men and women. J Strength Cond Res, 2007; 21: 
204-207

Chromiak JA, Smedley B, Carpenter W, Brown R, Koh YS, Lamberth JG, Joe LA, Abadie BR, Altorfer G. Effect of a 10-week strength training program and recovery drink on body composition, muscular strength and endurance, and anaerobic power and capacity. Nutrition, 2004; 20: 420-427

Craig CL, Marshall AL, Sjöström M, Bauman AE, Booth ML, Ainsworth BE, Pratt M, Ekelund U, Yngve A, Sallis JF, Oja P. International physical activity questionnaire: 12-country reliability and validity. Med Sci Sports Exerc, 2003; 35: 1381-1395

Fatouros IG, Kambas A, Katrabasas I, Nikolaidis K, Chatizinikolaou A, Leontsini D, Taxidaris K. Strength training and detraining effects on muscular strength, anaerobic power, and mobility of inactive older men are intensity dependent. Br J of Sports Med, 2005; 39: 776-780

Fitzgerald SJ, Barlow CE, Kampert JB, Morrow JR, Jackson AW, Blair SN. Muscular fitness and all-cause mortality: prospective observations. J Phys Act Health, 2004; 1: 7-18

Gleeson TT. Post-exercise lactate metabolism: a comparative review of sites, pathways, and regulation. Annu Rev Physiol, 1996; 58: 565-581

Haskell WL, Lee IM, Pate RR, Powell KE, Blair SN, Franklin BA, Macera CA, Heath GW, Thompson PD, Bauman A. Physical activity and public health: Updated recommendation for adults from the American College of Sports Medicine and the American Heart Association. Med Sci Sports Exerc, 2007; 39: $1423-1434$

Hunter GR, McCarthy JP, Bamman MM. Effects of resistance training on older adults. Sports Med, 2004; 3: 329-348

Inbar O, Bar-Or O, Skinner JS. The Wingate Anaerobic Test. Champaign IL: Human Kinetics, 1-40; 1996

Kim J, Cho HC, Jung HS, Yoon JD. Influence of performance level on anaerobic power and body composition in elite male judoists. J Strength Cond Res, 2011; 25: 1346-1354

Krieger JW. Single vs. multiple sets of resistance exercise for muscle hypertrophy: A meta-analysis. J Strength Cond Res, 2011; 24: 1150-1159

Liu-Ambrose T, Nagamatsu LS, Graf P, Beattie BL, Ashe MC, Handy TC. Resistance training and executive functions: A 12-month randomized controlled trial. Arch Intern Med, 2010; 170: 170-178

MacDougall JD, Ward GR, Sale DG, Sutton JR. Biochemical adaptation of human skeletal muscle to heavy resistance training and immobilization. J Appl Physiol Respir Environ Exerc Physiol, 1977; 43: 700-703

Macfarlane DJ, Taylor LH, Cuddihy TF. Very short intermittent vs continuous bouts of activity in sedentary adults. Prev Med, 2006; 43: 332-336

Miyashita M, Burns SF, Stensel DJ. Accumulating short bouts of brisk walking reduces postprandial plasma triacylglycerol concentrations and resting blood pressure in healthy young men. Am J Clin Nutr, 2008; 88: 1225-1231

Moraes E, Fleck SJ, Ricardo Dias M, Simão R. Effects on strength, power, and flexibility in adolescents of nonperiodized vs. daily nonlinear periodized weight training. J Strength Cond Res, 2013; 27: 3310-3321

Ng CL, Tai ES, Goh SY, Wee HL. Health status of older adults with Type 2 diabetes mellitus after aerobic or resistance training: A randomized trial. Health Qual Life Outcomes, 2011; 9: 1-5

O'Donovan G, Blazevich AJ, Boreham C, Cooper AR, Crank H, Ekelund U, Fox KR, Gately P, Giles-Corti B, Gill JMR, Hamer M, McDermott I, Murphy M, Mutrie N, Reilly JJ, Saxton JM, Stamatakis E. The ABC of Physical Activity for Health: A consensus statement from the British Association of Sport and Exercise Sciences. J Sports Sci, 2010; 28: 573-591

Oliveira AS, Gonçalves M. Neuromuscular recovery of the biceps brachii Muscle after resistance exercise. Res Sports Med, 2008; 16: 244-256

Reichert FF, Barros AJ, Domingues MR, Hallal PC. The role of perceived personal barriers to engagement in 
leisure-time physical activity. Am J of Public Health, 2007; 97: 515-519

Rabelo HT, Bezerra LA, Terra DF, Lima RM, Silva MA, Leite TK, de Oliveira RJ. Effects of 24 weeks of progressive resistance training on knee extensors peak torque and fat-free mass in older women. The effects of resistance training on explosive strength indicators in adolescent basketball players. $J$ Strength Cond Res, 2011; 25: 2298-2303

Reeves ND, Narici MV, Maganaris CN. In vivo human muscle structure and function: adaptations in resistance training in old age. Exp Physiol, 2004; 89: 675-689

Rimaud D, Messonnier L, Castells J, Devillard X, Calmels P. Effects of compression stockings during exercise and recovery on blood lactate kinetics. Eur J Appl Physiol, 2010; 110: 425-433

Santos EJ, Janeira MA. The effects of resistance training on explosive strength indicators in adolescent basketball players. J Strength Cond Res, 2012; 26: 2641-2647

Serwe KM, Swartz AM, Hart TL, Strath SJ. Effectiveness of long and short bout walking on increasing physical activity in women. J Womens Health, 2011; 20: 247-253

Slade JM, Miszko TA, Laity JH, Agrawal SK, Cress ME. Anaerobic power and physical function in strengthtrained and non-strength-trained older adults. J Gerontol A Biol Sci Med Sci, 2002; 57(3): M168-172

Tremblay MS, Warburton DE, Jansse, I, Paterson DH, Latimer AE, Rhodes RE, Kho ME, Hicks A, Leblanc AG, Zehr L, Murumets K, Duggan M. New Canadian physical Activity Guidelines. Appl Physiol Nutr Metab, 2011; 36: 36-46

Vanni AC, Meyer F, da Veiga AD, Zanardo VP. Comparison of the effects of two resistance training regimens on muscular and bone responses in premenopausal women. Osteoporosis Int, 2010; 21: $1537-1544$

Vardar SA, Tezel S, Oztürk L, Oktay K. The relationship between body composition and anaerobic performance of elite young wrestlers. J Sports Sci Med, 2007; 6: 34-38

Villanueva MG, Lane CJ, Schroeder ET. Short rest interval lengths between sets optimally enhance body composition and performance with 8 weeks of strength resistance training in older men. Eur J Appl Physiol, 2015; 115: 295-308

Washburn RA, Kirk EP, Smith BK, Honas JJ, Lecheminant JD, Bailey BW,

Donnelly JE. One set resistance training: Effect on body composition in overweight young adults. J Sports Med Phys Fitness, 2012; 52: 273-279

\section{Corresponding author:}

\section{Te Hung Tsao}

Physical Education Section of General Education,

National Sun Yat-Sen University,

70 Lienhai Rd., Kaohsiung 80424, Taiwan, R.O.C.

Tel: 886-7-7266553

Fax: 886-7-7266553

E-mail: t1208t2001@gmail.com 